\title{
Cooperative Control in Swarm Robotics Via Server
}

\author{
Judith R. Peters \\ BE (Computer Engg) \\ Sinhgad Academy of Engineering \\ Kondhwa (BK), Pune-48 \\ Yash S. Yawalkar \\ BE (Computer Engg) \\ Sinhgad Academy of Engineering \\ Kondhwa (BK), Pune-48
}

\author{
Saurabh S. Lokhande \\ BE (Computer Engg) \\ Sinhgad Academy of Engineering \\ Kondhwa (BK), Pune-48 \\ Bhushan S. Thakare \\ Professor (Computer Engg) \\ Sinhgad Academy of Engineering \\ Kondhwa (BK), Pune-48
}

\begin{abstract}
Swarm robots are a new approach in the robotic system. This idea of Swarm Robots is designed from the idea of small insects. Swarm robots can work co-ordinately and achieve its common goal. Self-organizing map (SOM)-based on the multi robots that have self-organizing criteria,Co-ordination and that can also be approached for dynamic environment. It can control number of robots and achieves different task locations randomly and gets its initial locations and directions.
\end{abstract}

\section{Keywords}

Co-operative control, Wi-Fi bot, Swarm Robotics, Swarm Intelligence, Computational Swarm Intelligence.

\section{INTRODUCTION}

Swarm robotics is becoming extremely interesting topic for multirobots researchers. The algorithm of swarm is mostly inspired by social behaviour of small animal and insects. Swarm robot is a concept of providing a robotics system by exploding large number of identical robots. The task is to design methods for enabling group of robots in the real world. Motion planning is considered to this kinds of robots. Efficiency and effectiveness is done by doing some studies. Swarm robots can fetch the given task by grouping together and completing the task. In this, robots have the task assignment and thus start when the tasks are given. These kinds of robots can achieve different task locations. The robots are cooperative to their task.

They have good interactions between them so that they can complete their given task. Due to their wireless property its connectivity range can extend, this can be the advantage of robots. The main aim of this study is to have

1.Co-operative control over the robots,

2.Efficient-working[12],

3.Self-organized[10],

4. Decision-making[4],

5. Task scheduling[12],

6. Co-ordination[18].

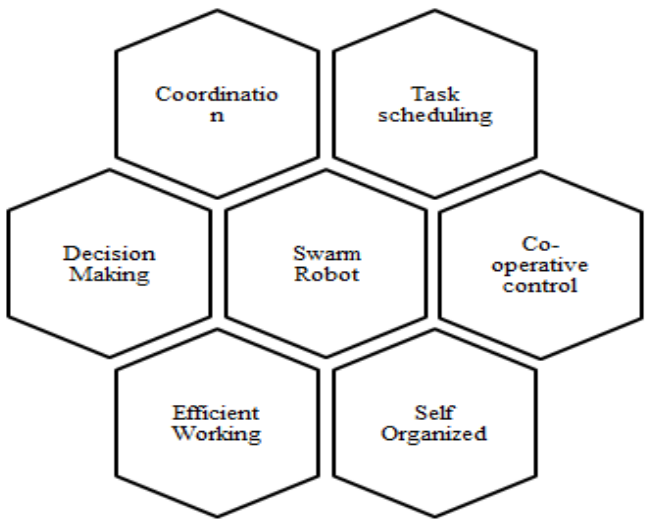

Fig 1. Main Aim.

Different task are divided to each of the bots and the targeted task is completed accordingly. So now it became easy to use these capabilities to locate the target. In this paper, we are creating small robots (bots) so that they can track down the target even in complex situations using ultrasonic sensors and can distribute its given task among the robots if the following robot is not capable of doing the task-alone

\section{ARCHITECTURE}

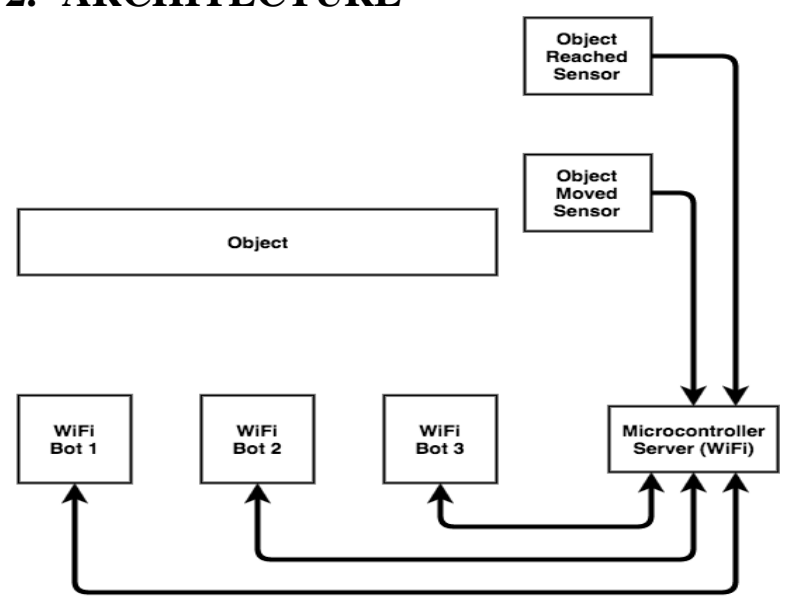

Fig2: Architecture

There are three bots constructed as shown in Fig2. Microcontroller server (Wifi) is connected with these bots. The bots have ultrasonic sensors so that it can track down the object. 


\section{WORKING}

As robotics is growing in this age, scientists continue to take some sort of information from the natural world, And now, those scientists are learning to use swarm intelligence to create robots. The main aim of this project is to study swarm robotics. Analyse its different parameters and focus on reducing the cost of design. Our initial aim is to design the autonomous robots. It means that if one robot is not able to push the object, it will communicate with the second robot and so on till the object reaches its destination.

\section{FLOWCHART}

\section{USE OF HARDWARE AND SOFTWARE 4.1 ESP8266 Wi-Fi Parameter:}

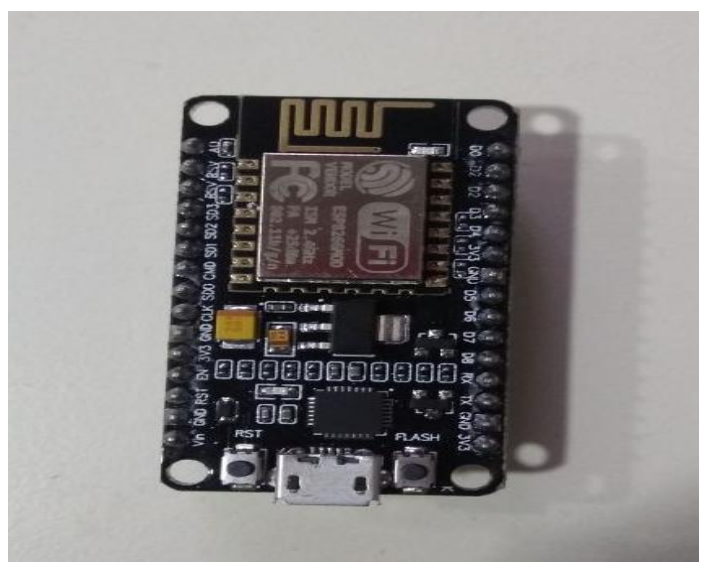

Fig 4: ESP2866 wifi parameter

- Wi-Fi protocols: $802.11 \mathrm{~b} / \mathrm{g} / \mathrm{n}$

- Frequency range: $2.4 \mathrm{G}$ to $2.5 \mathrm{G}(2400 \mathrm{M}$ to $2483.5 \mathrm{M})$ Hardware Parameters:

- Peripheral Bus: UART/SDIO/SPI/I2C/I2S/IR remote control

- Operating voltage: 3.0 to $3.6 \mathrm{~V}$ Software Parameters:

- WIFI mode: Station/softAP/softAP+Station

- Network Protocols: IPv4,TCP/UDP/HTTP/FTP

\subsection{ATMEGA328}

- Two 8 bit counter/timer

- Real Time counter with separate oscillators.

- 6 PWM channels

- 8 channel 10 bit ADC in TQFP package.

- Serial USART.

- On chip analog comparator.

\subsection{Ultrasonic sensors}

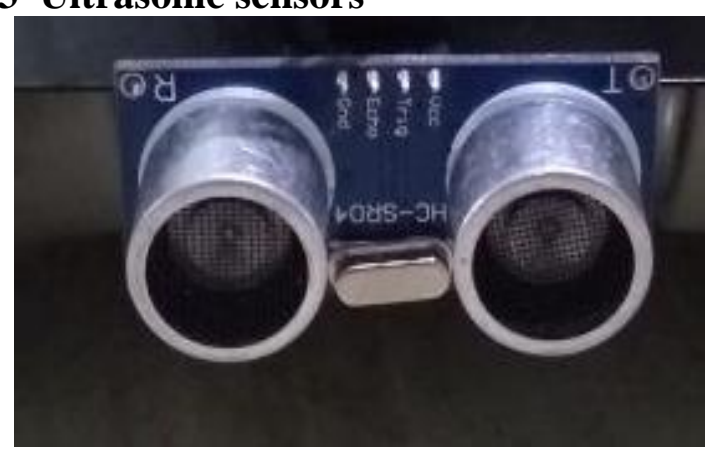

Fig 5: Ultrasonic sensors

\section{INFRARED RECEIVER}

- Quick acknowledgement time.

- $\quad$ Photo delicacy.

- Small juncture holding capacity.

- Operating temperature range: -40 to +85 .

\section{INFRARED TRANSMITTER}

- High reliability.

- High radiant intensity.

- $\quad 2.54 \mathrm{~mm}$ LED spacing.

- Low forward voltage.

\section{CALCULATIONS}

5.1 Hardware Design
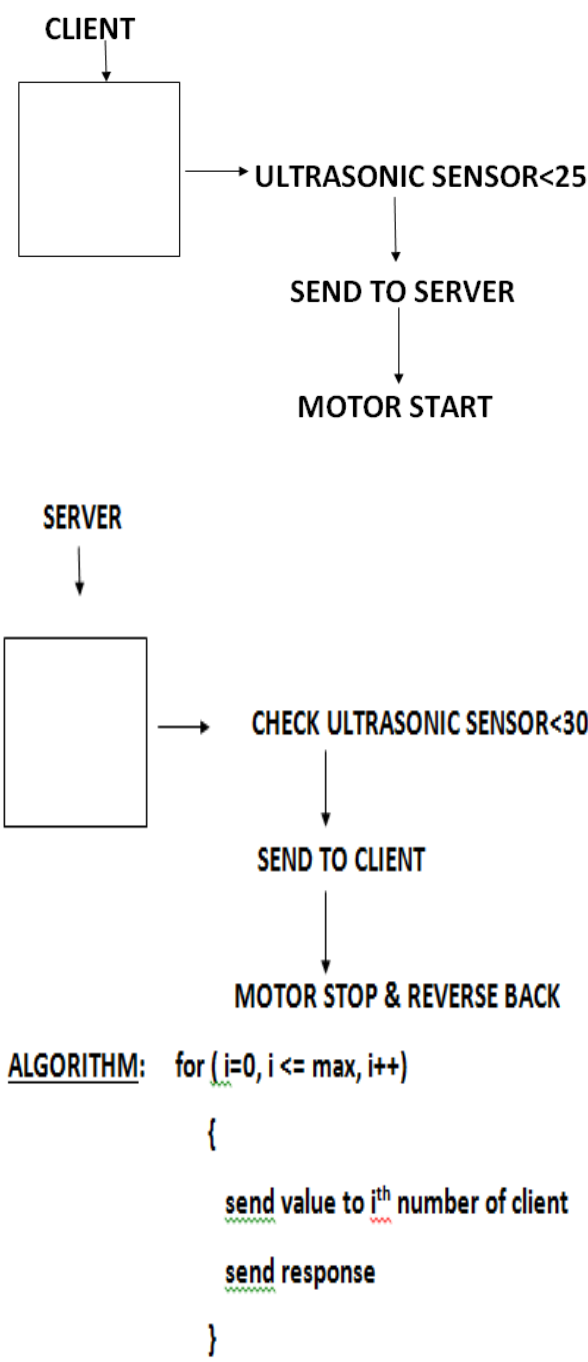

\section{OBSERVATIONS}

The client (bots) move according to the messages sent from server to the client

(bots) and visversa.

\section{Case 1:}

As the client(bot1) sense the object that is to be pushed to its destination,

within its $25 \mathrm{~cm}$ of area it moves towards the object 
and try to push it, when its able to push the object to its destination the work of the client is completed.

\section{Case 2:}

- If the client1(bot1) is not able to push the object the server sends the message to the client2(bot2) so as to push the object, along with client 1 .

\section{Case 3:}

If even the client2(bot2) is not able to push the object the server sends

message to the client3(bot3) to push the object along with client1,client2.

And hence, the pushing of object from its source to destination is done.

\section{RESULTS}

\subsection{Hardware Results:}

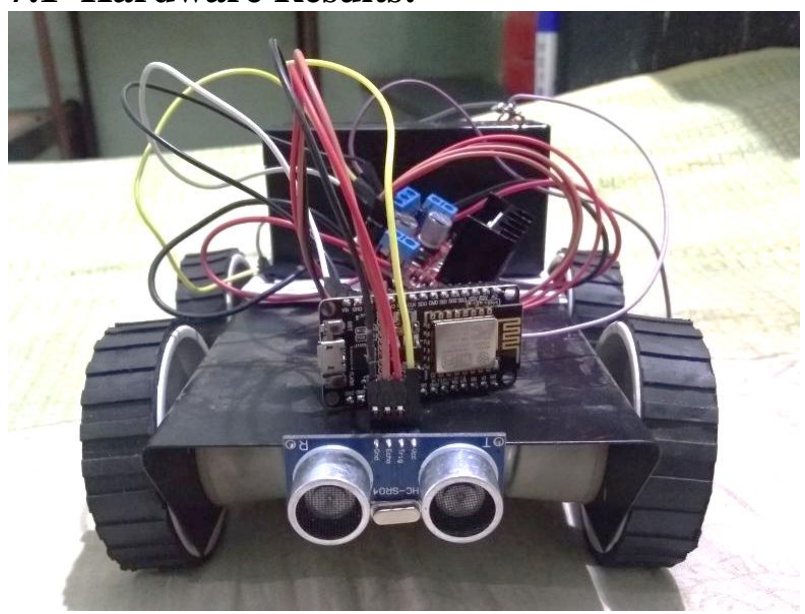

Fig 6: Front View(bot)

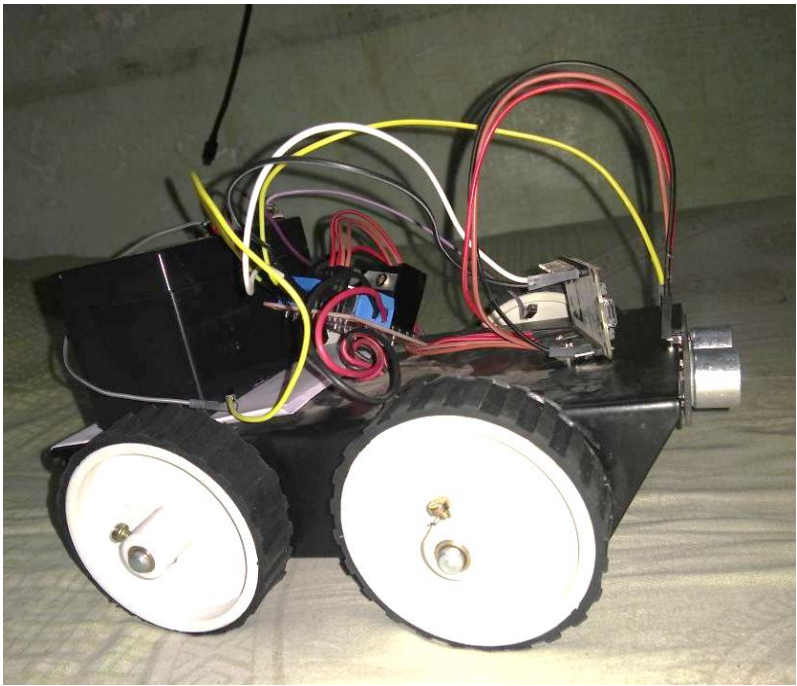

Fig 7: Side view(bot)

\section{CONCLUSION}

We have presented swarm based approach in cooperative control using server. The bots have to monitor the environment during specific time slots. Control and coordination is done by using swarm robotics. Can be able to use in military service for locating objects, automobile, mechanical fields etc. Self assembly of the robots are done as so it can coordinate with other bots and can provide stability to move the bots in unstructured terrain. Collectively transportation can be done by using bots.

\section{ACKNOWNLEGEMENT}

It gives us great pleasure presenting the project paper on 'COOPERATIVE CONTROL IN SWARM ROBOTICS VIA SERVER'. We would like to take this opportunity to thank our guide Prof. Bhushan Thakare for giving us all the help and guidance we needed.

\section{REFERENCES}

[1] Daniel Pickem, Paul Glotfelter, Li Wang, Mark Mote, Aaron Ames, Eric Feron, and Magnus Egerstedt "The Robotarium: A remotely accessible swarm robotics research testbed",2017 IEEE International Conference on Robotics and Automation (ICRA) Singapore, May 29 June 3, 2017.

[2] David Green, Jan Carlo Barca, Xinrang Wong, "Guideline for improving the robustness of swarm robotics system through adjustments of network",2017.

[3] Fernando J. Mendiburu, Marcos R. A. Morais, Antonio M.N. Lima, "Behavior coordination in multi-robot systems", IEEE 2016.

[4] Vasco Costa, Miguel Duarte, Tiago Rodrigues, Sancho Moura Oliveira and Anders Lyhne Christensen, "Design and Development of an Inexpensive Aquatic Swarm Robotics System”, Bio Machines Lab, Lisbon, Portugal, IEEE 2016

[5] R. Ramaya ,"Robotic Swarm Finding Shortest Path In am Unknow Environment", 2nd IEEE International Conference on Engineering and Technology (ICETECH), 17th\& 18thMarch 2016, Coimbatore, TN, India.

[6] Aaron Roggow, Georges El-Howayek,Sami Khorbotly,"Autonomous Identificatio of Local Agents in Multi-Agent Robotic Swarm.'IEEE 2016.

[7] Xin Yi, Anmin Zhu, Simon X. Yang, Chaomin Luo," A Bio-Inspired Approach to Task Assignment of Swarm Robots in 3-D Dynamic Environments",IEEE transaction on cybernetics, 2016.

[8] Mario Aguilera, Luis M. Torres-Trevino,"Change of Behavior on Swarm Robotics using Repulsion and attraction", Fourteenth Mexican International Conference on Artificial Intelligence,2015.

[9] Michail Papoutsidaki, Dimitrios Pirimalis, George Chamilothoris,"Co-operative mobile robotic platforms for wireless control applications",2014.

[10] Zhongyang Zheng, Jie Li, Junzhi Li and Ying Tan," Improved Group Explosion Strategy for Searching Multiple Targets using Swarm Robotics”,2014 IEEE International Conference on Systems, Man, and Cybernetics October 5-8, 2014, San Diego, CA, USA.

[11] Farshad Arvin, John C. Murray, Licheng Shi, Chun Zhang, Shigang Yue," Development of an Autonomous Micro Robot for Swarm Robotics", Proceedings of 2014 IEEE International Conference on Mechatronics and Automation August 3 - 6, Tianjin, China.

[12] Toshiyuki Yasuda, Kazuhiro Ohkura, Nanami Wada, and Yoshiyuki Matsumura," Behavior Sequence Analysis of Incrementally Evolving Robotic Swarms in a Foraging 
Task", Proceedings of the 2013 IEEE/SICE International Symposium on System Integration, Kobe International Conference Center, Kobe, Japan, December 15-17.

[13] Yara Khaluf,Emi Mathews, Franz J. Ramming,"SelfOrganized Co-operation in Swarm Robotics", 2011 14th IEEE International Symposium on Object/Component/Service-Oriented Real-Time Distributed Computing Workshops 2011.

[14] Peymon Gazi, Mo Jamshidi, Aleksandar Jevtic, Diego Andina, "A Mechatronic System Design Case Study: Control Of a Robotics Swarm Using Networked Control Algorithms.” 2010 IEEE.

[15] Aleksandar Jevtic,PaymonGazi, Diego Andina, Mo Jamshidi, "Building of Swarm of robotics bees"2010.

[16]Siddarth Jain, Manish Sawlani, Vijay Kumar Chandwani", A-hoc Swarm Robotics Optimization in Grid based Navigation”, 2010 11th Int. Conf. Control, Automation, Robotics and Vision Singapore, 7-10th December 2010

[17] Anmin Zhu, Simon X. Yang, "An improved SOMbased Approach to Dynamic Task Assignment of Multirobots", Proceedings of the $8^{\text {th }}$ World Congress on Intelligent Control and Automation July 6-9 2010, Jinan, China.
[18] Vitto Trianni, Stefano Nolfi, "Self- Organizing Sync in a Robotic Swarm: A Dynamical System View", IEEE transaction on evolutionary computation, VOL.13, NO. 4, AUGUST 2009

[19] Aleksandar Jevtic, Diego Andina," Swarm Intelligence and Its Applications in Swarm Robotics”, 2007.

[20] Marco Dorigo," Swarm Bot: An experiment in swarm robotics", Belgium, 2005.

\section{AUTHORS}

First Author- Judith R. Peters, BE(Computer Engg), Sinhgad Academy Of Engineering ,Kondhwa (BK), Pune-48 judithpeters07@gmail.com

Second Author- Saurabh S. Lokhande, BE(Computer Engg), Sinhgad Academy Of Engineering ,Kondhwa (BK), Pune-48 saurabhlokhande9@gmail.com

Third Author- Yash S. Yawalkar BE(Computer Engg), Sinhgad Academy Of Engineering ,Kondhwa (BK), Pune-48 yashsyawalkar@gmail.com

Fourth Author- Prof.Bhushan S. Thakare

(Computer Engg), Sinhgad Academy Of Engineering ,Kondhwa (BK), Pune-48

bsthakare.sae@sinhgad.edu 